\title{
REPARATION EN URGENCE DIFFEREE DES RUPTURES TRAUMATIQUES DE L'URETHRE POSTERIEUR
}

\author{
S.M. GUEYE, M. BA, C. SYLLA, A. NDOYE, B.A. DIAGNE, A. MENSAH
}

Service d'Urologie (Pr. A. MENSAH), CHU Aristide Le Dantec

Avenue Pasteur, DAKAR-SENEGAL

\section{DEFERATED EMERGENCY REPAIR OF THE DISRUPTED POSTERIOR}

URETHRA. The management of posterior urethra injuries is still subject of controversies. Some authors recommend primary or deferated emergency repair operation, while some others wait for about three months and repair the urethral stenosis. The analysis of a series of fifteen cases shows that when patients are treated by deferated emergency operation; the results are usually good. However, when repair is performed later, results are less good, and the rate of sexual impotency is higher in this group. We conclude that deferated emergency repair operation is the best way to manage disrupted posterior urethra. Key words : Posterior uethra injuries, deferated emergency repair. Andrologie, 1992, 2 : 121-122.

La rupture traumatique de l'urèthre postérieur, complication fréquente des traumatismes du bassin, est une lésion potentiellement grave. Cette gravité est liée à l'uropathie obstructive par sténose secondaire de l'urèthre, à l'incontinence urinaire,et à l'impuissance sexuelle. Son traitement fait encore l'objet de nombreuses controverses entre les partisans de la réparation immédiate, ceux de la réparation tardive au stade de sténose urétrale, et enfin,ceux qui prônent la réparation en urgence différée. Les premiers gestes déterminent le pronostic, d'où la nécessité d'une conduite thérapeutique adaptée gage d'une guérison sans séquelles.

\section{PATIENTS ET METHODES}

Cette étude rétrospective porte sur 15 cas de rupture traumatique de l'urèthre colligés en 4 ans. L'âge moyen des malades est de 22 ans avec des extrêmes de 13 et 44 ans.

Sur le plan clinique, les symptômes classiques (urétrorragie,impossibilité mictionnelle,ou hématome périnéal),ont toujours été retrouvés isolés ou diversement associés. Le diagnostic a été confirmé 8 fois par la cystographie directe ,et 7 fois par l'urographie intra-veineuse. La lésion siégeait au niveau de l'urèthre membraneux. dans tous les cas. Sept malades sont arrivés tardivement avec une sténose de l'urèthre déjà constituée. Ils ont tous bénéficié d'une urétroplastie. Les procédés utilisés ont été : lambeau cutané pénien de Quartey (4 cas), lambeau cutané pédiculé périnéal de Blandy (1 cas), urétroplastie simplifiée de Mickalowski ( 2 cas ). Les huit malades arrivés dans les suites immédiates du traumatisme ont été traités en urgence différée après drainage des urines par cathéter sus-pubien pendant 8 à 10 jours. Les résultats ont été évalués selon la qualité de la miction et des rapports sexuels postopératoires. Le recul moyen est de 3 ans.

\section{RESULTATS}

Sur le plan mictionnel (tableau $n^{\circ} 1$ )

Tableau $n^{\circ} 1$ : Résultats mictionnels

\begin{tabular}{|lccc|}
\hline & Urgence différée & Urétroplastie & TOTAL \\
\hline Bons & 6 & 1 & 7 \\
Moyens & 2 & 3 & 5 \\
Mauvais & - & 3 & 3 \\
\hline TOTAL & 8 & 7 & 15 \\
\hline
\end{tabular}

Ils ont été cotés bon, moyen ou mauvais, selon la qualité du jet mictionnel, la nécessité ou non d'une dilatation urétrale post-opératoire, ou bien d'une urétroplastie itérative. Parmi les malades opérés en urgence différée, six ont eu un bon débit mictionnel, tandis que chez 2 d'entre eux, un cathétérisme dilatateur (I à $2 /$ an) permet de maintenir un calibre urétral correct. Dans le groupe de malades traités pour sténose de l'urèthre,un seul malade a eu un bon résultat après uréthroplastie, trois malades ont eu besoin de dilatations rapprochées pour maintenir un bon jet mictionnel, et trois malades sont en cystostomie prolongée malgrè des urétroplasties itératives. Aucun malade ne se plaint d' incontinence urinaire.

\section{Sur le plan sexuel}

Quatre malades vus au stade de sténose urétrale, se plaignent d'une impuissance sexuelle post-traumatique.

\section{DISCUSSION}

Les controverses thérapeutiques soulevées par les ruptures traumatiques de l'urèthre postérieur découlent de la complexité pathogénique de certaines lésions en particulier génitales. L'hématome pelvi-périnéal, s'il n'est pas évacué, va s'organiser, devenir fibreux et constituer un callus responsable de la sténose urétrale. Aussi, en cas de fracture du pubis avec déplacement, la prostate qui reste attachée par les ligaments pubo-prostatiques, remonte dans le pelvis exagérant ainsi l'écart entre les deux moignons de l'urèthre rompu,et l'étendue de la sténose urétrale secondaire. Ainsi, certains auteurs $(3,5,9)$ ont préconisé un réalignement précoce de l'urèthre rompu, soit endoscopique avec mise en place d'une sonde urétrale tutrice,soit à ciel ouvert et anastomose termino-terminale. Les résultats mictionnels sont assez bons(tableau $n^{\circ} 2$ ), mais le taux d'impuissance sexuelle et d'incontinence parait très élevé.

Tableau ${ }^{\circ} 2$ : Résultats de la réparation immédiate

\begin{tabular}{|l|c|ccc|c|}
\hline & \multirow{2}{*}{ cas } & \multicolumn{3}{|c|}{ RESULTATS } & \% bons \\
& & Bon & Moyen & Echec & résultats \\
\hline Enquête A.F.C., 1980 & 26 & 9 & 10 & 7 & 35 \\
Filimon, 1981 & 10 & 10 & - & - & 100 \\
Viville, 1984 & 3 & 3 & - & - & - \\
Ridoux-Lobel, 1984 & 20 & 14 & - & 6 & 70 \\
Faure, 1984 & 6 & 3 & - & 6 & - \\
\hline TOTAL & $\mathbf{6 5}$ & $\mathbf{3 9}$ & $\mathbf{1 0}$ & $\mathbf{1 6}$ & - \\
\hline
\end{tabular}

Webster et coll.( 10) comparant deux groupes de malades provenant de 15 séries de rupture traumatique de l'urèthre postérieur, trouvent sur un total de 301 patients chez lesquels un réalignement précoce a été réalisé, des taux d'incontinence et d' impuissance de $20 \%$ et $44 \%$. Tandis que sur 236 patients opérés au stade de sténose urétrale, les taux respectifs d'incontinence et d'impuis-sance sexuelle sont de 1,7 et 11,6\%. Par contre, selon Turner-Warwick (8), le réalignement simple de l'urèthre sans dissection ni anastomose, n'aggrave pas le risque d'impuissance sexuelle. Nous pensons comme lui que l'impuissance sexuelle et l'incontinence ne sont pas toujours iatrogènes,comme il semble ressortir de l'étude de Webster, mais peuvent être le fait du traumatisme pelvien. En effet, les pédicules 
neuro-vasculaires destinés aux corps caverneux passent en dehors et en arrière de la prostate,et longent latéralement l'urèthre membraneux ; d'où la possibilité de leur lésion dans les ruptures de l'urètre postérieur.

L'impuissance sexuelle est très fréquente (tableau 2), et est souvent attribuée par les malades à l'intervention. Ainsi, certains auteurs,du fait des problémes médico-légaux préfèrent poser un cathéter sus-pubien et différer la réparation de l'urètre 3 à 6 mois plus tard. Pour notre part, nous ne pouvons justifier le choix délibéré d'attendre qu'une sténose urétrale se constitue avant d'envisager le traitement, dont on connait la difficulté et le caractère très aléatoire des résultats, malgrè les procédés d'urétroplasties par lambeaux cutanés pédiculés. En effet, même si les résultats initiaux sont souvent satisfaisants, ils se détériorent progressivement dans le temps justifiant des urétroplasties itératives. A notre avis, cette attitude n'est licite que dans un contexte polytraumatique, quand d'autres lésions priment sur l'urètre et qu'il faille tout juste dériver les urines par un cathéter sus-pubien et différer la réparation de l'urèthre rompu.

La réparation en urgence différée, à laquelle va notre préférence, est un moyen terme entre la réparation immédiate et la réparation tardive. Elle consiste à mettre en place un cathéter vésical suspubien par lequel nous pratiquons la cystographie. Cet examen permet de confirmer le diagnostic, de préciser le siège de la rupture et son caractère partiel ou complet. Le sondage urétral, dans un but diagnostic,doit être formellement proscrit $(2,3,6,7,8)$. Il risque de compléter une rupture partielle ,et surtout d'infecter le foyer traumatique.

La réparation de l'urèthre rompu se fera entre les huitième et dixième jours. En ce moment, l'hémostase spontanée s'est faite, l'hématome ne s'est pas encore organisé et est facile à évacuer. La vitalité des extrêmités de l'urèthre est facile à apprécier, ce qui permet de minimiser les risques de lachage des sutures, et de fistules post-opératoires. La réparation est facilitée par l'abord par voie trans-symphysaire, qui donne un excellent jour sur l'urèthre postérieur.

Tableau $n^{\circ} 3$ : Réparation en urgence différée

\begin{tabular}{|c|c|c|c|c|c|}
\hline & \multirow{2}{*}{ CAS } & \multicolumn{3}{|c|}{ RESULTATS } & \multirow{2}{*}{$\begin{array}{l}\% \text { bons } \\
\text { résultats }\end{array}$} \\
\hline & & Bon & Moyen & Echec & \\
\hline Enquête A.F.C., 1980 & 51 & 42 & 6 & 3 & 82 \\
\hline Faure, 1984 & 10 & 10 & 1 & I & 83 \\
\hline Bouchot, 1989 & 12 & 8 & 1 & 3 & 67 \\
\hline Notre série & 8 & 6 & 2 & - & - \\
\hline
\end{tabular}

Les bons résultats rapportés dans la littérature (tableau $n^{\circ} 3$ ) de même que notre expérience, plaident en faveur de la réparation en urgence différée des ruptures traumatiques de l'urèthre postérieur.

\section{REFERENCES}

1- Bouchot O., Guillonneau B., Karam G., Le Normand L., Buzelin J.M., Auvigne J. Les traumatismes de l'urètre postérieur. A propos de 57 patients, à l'exclusion des traumatismes iatrogènes. Ann. Urol., 1989, 23 : 220-226.

2 - Chatelain C. Ruptures traumatiques de l'urèthre : premiers gestes et lésions latrogenes. J. Urol., 1984, 90, n 10, PP.

3 - Devine C. J., Jordan G.H., Devine P.C. Primary realignment of the disrupted prostatomembranous urethra Urol. Clin. North America, 1989, 16.

4 - Faure G., Davin J.L., Rambeaud J., Revel M. Ruptures récentes de l'urèthre. De leur diagnostic à leur traitement. J. Urol. 1984, 90, $3: 187-190$.

5 - Filimon C. et coll. Réparation précoce des ruptures traumatiques de l'urètre postérieur chez l'homme : A propos de dix cas. J. Urol., Paris, 1981,$87 ; 419-426$

6 - Le Guillou M., Ferriere J.M. Traumatismes de l'urètre postérieur. Encycl. Med Chir, Paris, Reins-organes génito-urinaires, 1983, 18340 A 10-2.
7 - Moulonguet A. Points sur le traitement préconisé dans les traumatismes récents de l'urètre au moment du rapport de 1965. Ce qu'il en reste. Ce qui devrait changer. J. Urol., Paris, 1984,90, 659-660

8 - Turner-Warwick R. Prevention of complications resulting from pelvic fracture urethral injuries, and from their management. Urol. Clin. North America, 1989, 16.

9 - Viville Ch., Weltzer J., Bittar S., de Petronik. Quelques réflexions sur le diagnostic et le traitement d'urgence des ruptures traumatiques de l'urètre masculin dans les fractures du bassin. J. Urol., Paris, 1984, $90: 181-185$

10 - Webster G.D., Mathes G., Selli C. Prostatomembranous urethral injuries: A review of the litterature and a rational approach to their management. J. Urol., Boston, 1983, 130 : 898-902.

RESUME : Le traitement des ruptures traumatiques de l'urètre postérieur reste sujet à de nombreuses controverses entre les partisans de la réparation précoce en urgence soit immédiate, soit différée et ceux devenus plus rares qui prônent la réparation tardive au stade de sténose urétrale. De l'analyse d'une série de 15 cas colligés en 4 ans, il ressort que lorsque les malades sont pris en charge dans les suites immédiates de l'accident et traités en urgence différée, les résultats sont habituellement satisfaisants. Par contre chez les malades vus tardivement les résultats sont plus aléatoires sur le plan mictionnel malgrè des urétroplasties itératives. L'incidence de l'impuissance sexuelle semble plus élevée dans ce groupe de patients. Ces résultats corroborent ceux d'autres auteurs et plaident en faveur de la réparation en urgence différée des ruptures traumatiques de l'urèthre postérieur. Mots clés : Rupture urèthre postérieur, Urgence différée. Andrologie, 1992, 2 : 121-122. 